\title{
VARIOUS RIGGI IDENTITIES IN FINSLER SPACE
}

\author{
H. D. PANDE *
}

(Received 9 June 1967)

Ricci identities in a Finsler space have been given by C. I. Ispas [I], H. Rund [2], R. S. Mishra and H. D. Pande [3] and others. Here we shall prove some identities using the principle of mathematical induction. Considering $T^{i j}(x, \dot{x})$ a second order contravariant tensor depending on the element of support $\left(x^{i}, \dot{x}^{i}\right)$, we have the following theorems.

\section{Riccl identities involving the Cartan's first type of covariant derivative}

THEOREM 1.1. The Ricci identity for a contravariant tensor $T^{i j}(x, \dot{x})$ of order two is given by

$$
T^{i j}{ }_{\mid h k}-T^{i j}{ }_{\mid k h}=-\left.T^{i j}\right|_{\varepsilon} K_{r h k}^{s} t^{r}+T^{r j} R_{r h k}^{i}+T^{i r} R_{r h k}^{j},
$$

where $K_{r h k}^{s}(x, \dot{x})$ and $R_{r h k}^{s}(x, \dot{x})$ are the curvature tensors and the symbol I followed by an index denotes the Cartan's second type of covariant derivative [2].

Proof. Let $X^{i}(x, \dot{x})$ and $B_{s}(x, \dot{x})$ be the contravariant and covariant components of two vector fields. We have [2]

$$
X^{i}{ }_{\mid k k}-X^{i}{ }_{\mid k h}=R_{j h k}^{i} X^{j}-\left.K_{r h k}^{j} t^{r} X^{i}\right|_{j},
$$

and

$$
B_{i \mid h k}-B_{i \mid k h}=-B_{r} R_{i h k}^{r}-\left.B_{i}\right|_{z} K_{r k k}^{l} t^{r},
$$

where $t^{r}$ is the unit tangent vector.

Let $B_{j}(x, \dot{x})$ be an arbitrary covariant vector field such that its inner product with the tensor $T^{i j}(x, \dot{x})$ is given by

$$
X^{i}(x, \dot{x}) \stackrel{\text { def }}{=} T^{i j}(x, \dot{x}) B_{j}(x, \dot{x})
$$

Eliminating $X^{i}(x, \dot{x})$ from (1.2) and (1.4) and using (1.3), we get

$$
B_{j}\left[T^{i j}{ }_{\mid h k}-T^{i j}{ }_{\mid k h}+\left.T^{i j}\right|_{8} K_{r h k}^{s} t^{r}-T^{r j} R_{r h k}^{i}-T^{i r} R_{r h k}^{j}\right]=0 .
$$

* At present with the Department of Mathematics, University of Western Australia, Nedlands, W.A. 
Since $B^{j}(x, \dot{x})$ is an arbitrary vector, the formula follows from the equation (1.5).

THEOREM 1.2. The Ricci identity for a contravariant tensor $T^{j_{1}} \cdots, j_{e}(x, \dot{x})$ of order $q$ is given by

$$
\begin{aligned}
& T^{j_{1}, \cdots, j_{{ }_{\mid h k}}}-T^{j_{1}, \cdots, j_{a_{\mid k h}}}=-\left.T^{j_{1}, \cdots, j_{q}}\right|_{g} K_{r h k}^{s} t^{r}
\end{aligned}
$$

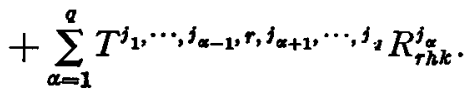

Proof. Let us suppose that the identity is true for a contravariant tensor of order, say, $m(<q)$. Thus we have

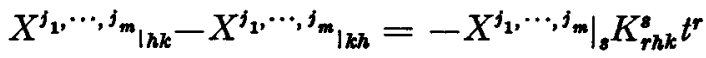

$$
\begin{aligned}
& +\sum_{\beta=1}^{m} X^{j_{1}, \cdots, j_{\beta-1}, r, j_{\beta+1}, \cdots, j_{m}} R_{r h k}^{j_{\beta} \beta} \text {. }
\end{aligned}
$$

The inner product of an $(m+1)$ th order contravariant tensor $T^{j_{1}, \cdots, j_{m}, i}(x, \dot{x})$ with an arbitrary covariant vector $B_{i}(x, \dot{x})$ is given by

$$
X^{j_{1}}, \cdots, j_{m}(x, \dot{x}) \stackrel{\text { def }}{=} T^{j_{1}}, \cdots, j_{m}, i(x, \dot{x}) B_{i}(x, \dot{x})
$$

Eliminating $X^{j_{1}, \cdots, j_{m}}(x, \dot{x})$ from (1.7) and (1.8) and using (1.3), we obtain

$$
\begin{aligned}
B_{i}\left[T^{j_{1}, \cdots, j_{m}, i}{ }_{1 h k}\right. & -T^{j_{1}, \cdots, j_{m}, i}{ }_{\mid k h h} \\
& +\left.T^{j_{1}, \cdots, j_{m},{ }^{i}}\right|_{s} K_{r h k}^{s} t^{r}-T^{j_{1}, \cdots, j_{m}, r} R_{r h k}^{i} \\
& \left.-\sum_{\beta=1}^{m} T^{j_{1}, \cdots, j_{\beta-1}, r, j_{\beta+1}, \cdots, j_{m}, i} R_{r h k}^{j_{k}}\right]=0 .
\end{aligned}
$$

Since $B_{i}(x, \dot{x})$ is an arbitrary vector field, we may replace the index $i$ by $j_{m+1}$ in the above equation to get

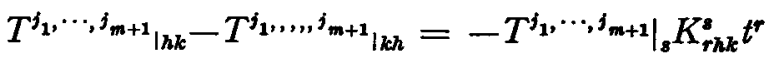

$$
\begin{aligned}
& +\sum_{\alpha=1}^{m+1} T_{1}^{j_{1}, \cdots, j_{\alpha-1}, r, j_{\alpha+1}, \cdots, j_{m+1}} R_{r h k}^{j_{k}} \text {. }
\end{aligned}
$$

Hence, by induction, the theorem holds.

\section{Ricci identities involving the Cartan's second type of covariant derivative}

THEOREM 2.1. The Ricci identity for a contravariant tensor $T^{i j}(x, \dot{x})$ of order two is given by

$$
\left.T^{i j}\right|_{h k}-\left.T^{i j}\right|_{k h}=\left\{\left.F_{t^{k}} T^{i j}\right|_{h}-\left.F_{t^{k}} T^{i j}\right|_{k}\right\}+T^{i r} S_{r k h}^{j}+T^{r j} S_{r k h}^{i},
$$

where $S_{r k h}^{i}(x, \dot{x})$ are the Cartan's first curvature tensor [2] and $F_{\dot{x}^{k}} \stackrel{\text { def }}{=} \partial F / \partial \dot{x}^{k}$.

Proof. Let $X^{i}(x, \dot{x})$ and $B_{j}(x, \dot{x})$ be the contravariant and covariant components of two vector fields. We have [2] 


$$
\left.X^{i}\right|_{h k}-\left.X^{i}\right|_{k h}=\left\{\left.F_{\dot{x}^{k}} X^{i}\right|_{h}-\left.F_{\dot{x}^{\wedge}} X^{i}\right|_{k}\right\}+S_{j k h}^{i} X^{j},
$$

and

$$
\left.B_{i}\right|_{h k}-\left.B_{i}\right|_{k h}=\left\{\left.F_{\dot{x}^{k}} B_{i}\right|_{h}-\left.F_{\dot{x}^{h}} B_{i}\right|_{k}\right\}-B_{j} S_{i k h}^{j} .
$$

Eliminating $X^{i}(x, \dot{x})$ from (1.4) and (2.2) and using (2.3), we obtain

$$
B_{j}\left[\left.T^{i j}\right|_{h k}-\left.T^{i j}\right|_{k h}-\left\{\left.F_{x^{k}} T^{i j}\right|_{h}-\left.F_{\dot{x}^{k}} T^{i j}\right|_{k}\right\}-T^{i r} S_{r k h}^{j}-T^{r j} S_{r k h}^{i}\right]=0 .
$$

Since $B_{j}(x, \dot{x})$ is an arbitrary covariant vector, theorem 2.1 follows from the above equation.

Theorem 2.2. The Ricci identity for a contravariant tensor $T^{i_{1}} \cdots, j_{a}(x, \dot{x})$ of arbitrary rank $q$, say, is given by

$$
\begin{aligned}
T^{j_{1}} \cdots,\left.{ }_{q}\right|_{h k}-\left.T^{j_{1}, \cdots, j_{q}}\right|_{k h}= & \left\{F_{\dot{x}^{k}} T^{j_{1}}, \cdots,\left.j_{q}\right|_{h}-F_{\dot{x}^{\wedge}} T^{j_{1}}, \cdots,\left.j_{q}\right|_{k}\right\} \\
& +\sum_{\beta=1}^{q} T^{j_{1}, \cdots, j_{\beta-1}, r, j_{\beta+1}, \cdots, j_{q}} S_{r k h}^{j_{\beta}} .
\end{aligned}
$$

Proof. Let the theorem be true for a contravariant tensor of order, say, $m(<q)$. Thus we have

$$
\begin{aligned}
\left.X^{j_{1}, \cdots, j_{m}}\right|_{h k}-X^{j_{1}, \cdots,\left.j_{m}\right|_{k h}=} & \left\{\left.F_{\alpha^{k}} X^{j_{1}, \cdots, j_{m}}\right|_{h}-F_{x^{h}} X^{j_{1}} \cdots,\left.j_{m}\right|_{k}\right\} \\
& +\sum_{\alpha=1}^{m} X^{j_{1}, \cdots, j_{\alpha-1}, r, j_{\alpha+1}} \cdots, j_{m} S_{r k h}^{j_{k}} .
\end{aligned}
$$

The inner product of an $(m+1)$ th order contravariant tensor $T^{j_{1}}, \cdots, j_{m}, i(x, \dot{x})$ with an arbitrary covariant vector field is defined by (1.8). Eliminating $X^{j_{1}, \cdots, j_{m}, i}(x, \dot{x})$ from (1.8) and (2.6) and using (1.3), we get

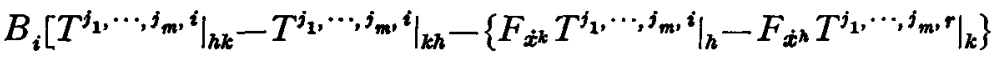

$$
\begin{aligned}
& \left.-\sum_{\alpha=1}^{m} T^{j_{1}, \cdots, j_{\alpha-1}, r, j_{\alpha+1}, \cdots, j_{m}, i} S_{r k h}^{j_{\alpha}}-T^{j_{1}, \cdots, j_{m}, i} S_{r k h}^{i}\right]=0 .
\end{aligned}
$$

Since $B_{i}(x, \dot{x})$ is an arbitrary vector field, we may replace the index $i$ by $j_{m+1}$ in the above equation to obtain

$$
\begin{aligned}
& \left.T^{j_{1}, \cdots, j_{m+1}}\right|_{h k}-T^{j_{1}}, \cdots,\left.j_{m+1}\right|_{k h}=\left\{\left.F_{\dot{x}^{k}} T^{j_{1}, \cdots, j_{m+1}}\right|_{h}-F_{\dot{x}^{h}} T^{j_{1}, \cdots,\left.j_{m+1}\right|_{k}}\right\} \\
& +\sum_{\alpha=1}^{m+1} T^{j_{1}}, \cdots, j_{\alpha-1}, r, j_{\alpha+1}, \cdots, j_{m+1} S_{r k h}^{j_{\alpha}} \text {. }
\end{aligned}
$$

Hence, by induction, the theorem holds.

\section{Ricci identities involving the Cartan's both type of covariant derivatives}

THEOREM 3.1. The Ricci identity for a contravariant tensor $T^{i j}(x, \dot{x})$ of order two is given by 


$$
\begin{aligned}
\left.T^{i j}\right|_{\left.h\right|_{k}}-\left.T^{i j}{ }_{\mid k}\right|_{h}= & -T^{i r} P_{r k h}^{j}-T^{r j} P_{r k h}^{i} \\
& +\left.T^{i j}\right|_{m} A_{k k \mid r}^{m} t^{r}+\left.T^{i j}\right|_{r} A_{h k,}^{r},
\end{aligned}
$$

where $t^{r}$ is the unit tangent vector and $P_{r k h}^{j}(x, \dot{x})$ are the Cartan's second curvature tensors [2].

Proof. Let $X^{i}(x, \dot{x})$ and $B_{j}(x, \dot{x})$ be the contravariant and covariant components of two vector fields. We have [2].

$$
\left.X^{i}\right|_{h \mid k}-\left.\left.X^{i}\right|_{k k}\right|_{h}=-X^{j} P_{j k h}^{i}+\left.X^{i}\right|_{j} A_{h k \mid r}^{j} t^{r}+X^{i}{ }_{j} A_{h k}^{j},
$$

and

$$
\left.B_{i}\right|_{h \mid k}-\left.B_{i \mid k}\right|_{h}=B_{j} P_{i k h}^{j}+\left.B_{i}\right|_{j} A_{\left.h k\right|_{r}}^{j} t^{r}+B_{\left.i\right|_{j}} A_{h k}^{j} .
$$

The inner product of an arbitrary covariant vector $B_{s}(x, \dot{x})$ with $T^{i j}(x, \dot{x})$ is given by (1.4). Eliminating $X^{i}(x, \dot{x})$ from (1.4) and (3.2) and using (3.3), we get

$$
\begin{aligned}
B_{j}\left[\left.T^{i j}\right|_{h \mid k}-\left.T^{i j}{ }_{\mid k}\right|_{h}+T^{i r} P_{r k h}^{j}+T^{r j} P_{r k h}^{i}\right. \\
\left.-\left.T^{i j}\right|_{m} A_{h k \mid r}^{m} t_{r}-T^{i j}{ }_{{ }_{r}} A_{h k}^{r}\right]=0 .
\end{aligned}
$$

Since $B_{3}(x, \dot{x})$ is an arbitrary vector field, we get the result (3.1).

THEOREM 3.2. The Ricci identity for a contravariant tensor $T^{j_{1}}, \cdots,{ }_{a}{ }_{a}(x, \dot{x})$ of arbitrary rank, say, $q$ is given by

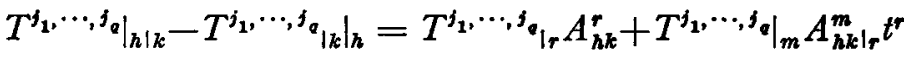

$$
\begin{aligned}
& -\sum_{\beta=1}^{q} T^{j_{1}} \cdots, j_{\beta-1}, r, j_{\beta+1}, \cdots, j_{e} P_{r k h}^{j_{\beta}} \text {. }
\end{aligned}
$$

Proof. Let the theorem be true for a contravariant tensor of order, say, $m(<q)$. Thus we have

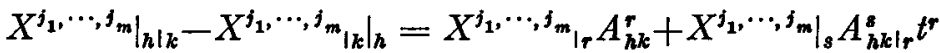

$$
\begin{aligned}
& +\sum_{\alpha=1}^{m} X^{j_{1}, \cdots, j_{\alpha-1}, r, j_{\alpha+1}, \cdots, j_{m}} P_{r k h}^{j_{\alpha}} \text {. }
\end{aligned}
$$

Eliminating $X^{j_{1}}, \cdots, j_{m}(x, \dot{x})$ from (1.8) and (3.6) and using (3.3), we get

$$
\begin{array}{r}
B_{i}\left[\left.T^{j_{1}, \cdots, j_{m}, i_{h}}\right|_{h 1 k}-\left.T^{j_{1}, \cdots, j_{m}, i}{ }_{\mid k}\right|_{h}-T^{j_{1}, \cdots, j_{m}, i}{ }_{\mid r} A_{h k}^{r}+\left.T^{j_{1}, \cdots, j_{m}, i_{3}}\right|_{s} A_{h k \mid r}^{s} t^{r}\right. \\
\left.+\sum_{\alpha=1}^{m} T^{j_{1}, \ldots, j_{\alpha-1}, r, j_{\alpha+1}, \cdots, j_{m}, i} P_{r k h}^{j_{\alpha}}+T^{j_{1}, \cdots, j_{m}, r} P_{r k h}^{i}\right]=0 .
\end{array}
$$

Since $B_{i}(x, \dot{x})$ is an arbitrary covariant vector field, we may replace the index $i$ by $i_{m+1}$ in the above equation to get 


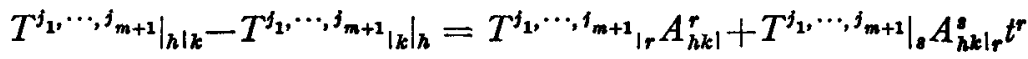

$$
\begin{aligned}
& +\sum_{\alpha=1}^{m+1} T^{j_{1}}, \cdots, j_{\alpha-1}, r, j_{\alpha+1}, \cdots, j_{m+1} P_{r k h}^{j_{\alpha}} \text {. }
\end{aligned}
$$

Hence, by induction, the theorem holds.

\section{Acknowledgement}

I would like to thank my supervisor Professor R. S. Mishra for his kind help in the preparation of this paper and the referee for his valuable suggestions.

\section{References}

[1] C. I. Ispas, 'Identite's de type Ricci dans l'éspace de Finsler', Com. Acad. R. P. Române 2 (1952), 13-18.

[2] H. Rund, The Differential Geometry of Finsler spaces (Springer Verlag, Berlin, 1959).

[3] R. S. Mishra and H. D. Pande. 'The Ricci identity', Annali di Matematica, pura ed applicata 75 (1967), 355-361.

[4] R. S. Mishara, A course in tensor with application to Riemannian Geometry (Pothishala Pvt. Ltd. Alld., India, 1965).

[5] R. B. Misra, Some problems in Finsler spaces (Ph.D. Thesis, University of Allahabad, 1967).

Department of Mathematics

University of Gorakhpur

Gorakhpur

India

$$
\text { and }
$$

Department of Mathematics

University of Western Australia

Nedlands, W.A. 